\title{
Application of Brody Growth Function to Describe Dynamics of Breast Cancer Cells
}

\author{
Abdulsamad Engida Sado, Purnachandra Rao Koya \\ School of Mathematical and Statistical Sciences, Hawassa University, Hawassa, Ethiopia
}

Email address:

samutiya@gmail.com (A. E. Sado),drkpraocecc@yahoo.co.in (P. R. Koya)

To cite this article:

Abdulsamad Engida Sado, Purnachandra Rao Koya. Application of Brody Growth Function to Describe Dynamics of Breast Cancer Cells. American Journal of Applied Mathematics. Vol. 3, No. 3, 2015, pp. 138-145. doi: 10.11648/j.ajam.20150303.20

\begin{abstract}
In this paper we have constructed a mathematical model using Brody function and applied to describe the dynamics of breast cancer. To construct the mathematical model we considered that the linear cancer network technique describes the growth of estrogen receptor positive breast cancers. Model validity is verified using simulation study and mathematical analysis. It is verified that the hormone therapy is a technique to treat endocrine receptor positive breast cancers. Hormone therapy is considered as a treatment and used to block the estrogens receptors from the cancer and health cells. Important observations are made from the simulation study and physical interpretations are drawn and presented lucidly in the paper.
\end{abstract}

Keywords: Koya - Goshu, Brody Model, Breast Cancer, Cancer Stem Cell, Cancer Tumor Cell, Hormone Therapy and Tamoxifen

\section{Introduction}

Cancer has been a major cause, among all the diseases, for human death throughout the world. An estimation of 14.10 million cancer cases of all types were found around the world in the year 2012, of those cases 7.40 million cases were found among men and 6.70 million cases in women. This number is expected to increase steadily and reach up to 24 million by the year 2035. Breast cancer cases in women occupy the second most in the list of common cancer types with nearly 1.7 million such new cases found in 2012. Breast cancer cases among women diagnosed in 2012 is about $11.9 \%$ of all types of cancer cases found in both men and women except nonmelanoma skin cancer. Among women breast cancer alone occupy more than $25 \%$ of the cumulative number of all types of the cases identified during2012worldwide.So far the developed countries have been experiencing about $55 \%$ of all the globally identified cancer cases but the cancer occurrence rates have started to rise rapidly among the developing countries due to globalization and other influencing factors [13-14].

In Ethiopia breast cancer is the second most occurring cancer among women which is next to the cervical cancer. It is estimated that around ten thousand Ethiopian women have been affected with breast cancer every year. However, thousands of cases go unreported because the rural women often approach traditional healers for treatment. A very few cases seek treatment from the government health organizations where only these cases are recorded $[3,6,9]$.

The cancer decease is a result of the uncontrolled growth of abnormal cells in human body. Uncontrolled growth of abnormal cells is a result of a set of mutations in the healthy cells. Cells are building blocks of the body and cancer starts from normal cells. Cells in the body born and die naturally. In order to balance natural death of the cells and to maintain cell population equilibrium state, the normal cells in human body divide themselves and grow in number. Cancer occurs when unbounded and fast growth of cells takes place in the body. As a whole, cancer is a collection of more than a hundred diseases or reasons in human body $[2,7]$.

A variety of cancer diseases can occur in human body. In general, name to the cancer disease is given with the name of the body part where the disease occurs viz., Brain cancer, Lung cancer, Bone cancer, blood cancer and Breast cancer are a few in the list. Any party of the human body can be affected by cancer disease $[2-3,5,10]$.Breast cancer is the most common cancer affecting women worldwide. The breast cancer in women can occur in many forms including 
Endocrine receptor positive for estrogen, Endocrine receptor negative for estrogen, Endocrine receptor positive for progesterone, Endocrine receptor negative for progesterone, HER2 positive and HER2 negative. The most common type of breast cancer in women is Endocrine receptor positive breast cancer. The rise of Estrogen hormone in women human body to the higher levels above the normal level causes an increase in the rate of cell growth and that leads to mutation in healthy cells that resulting in the birth of cancer cells.

The common signs of breast cancer include one or more of the following: (i) a hard compact and unshapely mass develop in the breast or armpit. This lump is painless and it occurs only on one side of the breast, (ii) the breast size increases and / or its shape changes. Indentation (concave shaped cuts on an edge) of breast occurs. Veins (pipes in the body through which blood circulates) grow. Skin erosion (be destroyed gradually) take place, (iii) various changes will appear in the skin. Skin becomes hard. Small hollows (dimpling) will occur in the skin. Swellings (bumps) occur. The color of the skin will change to redness or to orange, and (iv) various changes will appear in the nipple. Nipple goes back into the breast (retraction).Unusual liquids are produced and discharged around the nipple area. Breaking of skin causing red spots (rashes) will occur around the nipple area.

The treatment choices for breast cancer in women include radiation therapy, hormone therapy, targeted therapy, chemotherapy and surgery. Hormone therapy is mainly used to work against Hormone receptive positive breast cancer. Hormone therapy helps to lower the risk of reoccurring of breast cancer after surgery. Hormone therapy is applied to treat breast cancer very frequently. Recall that the high amount of estrogen causes breast cancer. Hormone therapy reduces estrogen levels and helps breast cancer to be under control. Hormone therapy effectively prevents the effect of estrogen on the breast cancer cells $[2,5,10]$.

The technique of anti-estrogens has been the first among all these therapies. This treatment method was very classical and adopted to fight against the breast cancer. Also for a long time the drug containing tamoxifen has been the standard front-line treatment to fight against the breast cancer that occurs after menopausal. Symptoms of menopause or temporary menopause may cause due to the hormonal treatments. Hormone therapy is effectively used in premenopausal and post-menopausal women and it works also well in the remission stage of the breast cancer $[2,7,10]$.

In Section 2, we have introduced the Brody growth function as a special case of Koya - Goshu generalized growth function. In Section 3, mathematical modeling to represent the dynamics of breast cancer cells is constructed using Brody growth function. Model variables and parameters are defined. In Section 4, simulation study with various sets of parametric values is taken up and important observations are interpreted. In Section 5, mathematical analysis of the model is considered. Equilibrium points are identified and their positivity and stability are discussed. The paper ends with concluding remarks in Section 6.

\section{Brody Model}

Brody growth model is the special case of Koya -Goshu generalized biological growth function where the latter is defined as $f(t)=A_{L}+\left(A-A_{L}\right)\left[1-B e^{-k\left([t-\mu / \delta)^{\psi}\right.}\right]^{m}$

Here the symbol $B=1-\left[\left(A_{\mu}-A_{L}\right) /\left(A-A_{L}\right)\right]^{\frac{1}{m}}$

Represents a notation and is derived from the four parameters $A, A_{L}, A_{\mu}$ and $m$. The eight parameters involved in the definition for the function are defined as follows: (i) $A=$ $\lim _{t \rightarrow \infty} f(t)=f_{\infty}$ where $A$ is the upper asymptote of $f(t)$, (ii) $A_{L}$ is the lower asymptote of $f(t)$, (iii) $A_{\mu}=f(\mu)$, (iv) $k$ is the growth rate parameter, (v) $\mu$ is the time shift parameter and is a constant, (vi) $\delta$ is time scale parameter and is a constant, and(vii) $v \neq 0$ and $m \neq 0$ a are the shape parameters. The Koya-Goshu growth model is an eight parameter $\left(A, A_{L}, A_{\mu}, k, \mu, \delta, v, m\right)$ function. The commonly used growth models such as Brody, Von Bertalanffy, Richards, Weibull, Monomolecular, Mitscherlich, Gompertz, Logistic and Generalized Logistic functions all are special cases of Koya-Goshu growth. The model is a more general solution of the rate-state equation represented by the first ordinary differential equation $[d f(t) / d t]=r_{t} f(t)$ and the detailed description is given in $[1,4]$. Brody function is a special case of Koya - Goshu growth function with the parameters taking the values. $A_{L}=0, \mu=0, \delta=1 v=1$, and $m=1$, However, Brody function (Brody, 1945) describing biological growths is defined by

$$
f(t)=A\left(1-B e^{k t}\right) \quad f(t)=A\left(1-B e^{-k t}\right)
$$

Here in (1) the parameters in their original notations represent: (i) $B=1-\left(A_{0} / A\right)$, (ii) $A=\lim _{t \rightarrow \infty} f(t)$ is upper asymptote of $f(t)$, (iii) $A_{0}=f(0)$ denotes growth at the initial point of time $t=0$, and (iv) $k$ represents the growth parameter $[1,4]$. In fact the Brody function given in (1) is a solution of the rate-state equation $[d f(t) / d t]=r_{t} f(t)$ where the relative rate function is given by $r_{t}=k[(A / f(t))-1]$. Thus, the rate-state equation for Brody function can be expressed as $d f(t) / d t=k A[1-(f(t) / A)]$, see [4].

\section{Brody Function to Represent Breast Cancer Cell Growth}

The most common type of breast cancer in women forms when estrogen levels in them are increased intensively. A sequence of reasons contribute for the disease formation and the flow contains the following: Increased levels of estrogen leads to increase in the rate of cellular, latter leads to mutation in health cells which in turn resulting in endocrine receptor positive breast cancer 
The idea of Eric Werner [8, 10] can be followed as a framework, in order to construct a mathematical model to represent and describe (i) growth of breast cancer cells and (ii) the effect of Hormone therapy treatment on breast cancer. We use Brody function to construct a set of two differential equations for modeling the growth of cancer stem cells and cancer tumor cells. Eric

It has been proposed a new design to represent cancer growth. This new design suggests that cancer develops as a result of mutations in the normal cells. The network of mutations describes the behavior of cancer stem cells. The stem cells divided and produce tumor cells depending on the instructions of the corresponding network. It is proposed to consider linear cancer networks in which the cancer stem cells can either be divided into cells of the same type or produce tumor cells. Once the model of linear cancer network is constructed, it can be extended to include the effect of estrogen presence and that of the Hormone therapy treatment $[8,10]$.

The number of cancer stem cells is represented by $A(t)$ while the number of cancer tumor cells is represented by $\mathrm{B}(\mathrm{t})$ respectively at any point of time $\mathrm{t}$. Further, to compute even before hand the effect of hormone therapy on both cancerous and healthy cells, a third differential equation that describes the growth rate of healthy cells within the breast tissue is required to be constructed. The number of healthy cells at any point of time is represented by $H(t)$ and we will use Brody function to model $H(t)$ too.

In our present study we consider Brody function as $f(t)=M\left(1-b e^{k t}\right)$ where the constant parameter $M=f(\infty)$ representing the caring capacity or upper asymptotic value or maximum value. The value of the constant $b$ can be computed using the formula $b=1-\left(M_{0} / M\right)$ where the constant $M_{0}=f(0)$ represents lower asymptotic value or minimum value. Also the analogues ratestate equation representing Brody function can be expressed as $d f(t) / d t=k M[1-(f(t) / M)]$. We now apply the Brody function in its form as described here to construct a

$$
\begin{gathered}
d A / d t=\left(k E / E_{0}\right) M_{1}\left[1-\left(A / M_{1}\right)\right]-d_{1}\left[1-\left(E / E_{0}\right)\right] A \\
d B / d t=\left(k E / E_{0}\right) A\left[1-\left(B / M_{2}\right)\right]-n B-d_{2}\left[1-\left(E / E_{0}\right)\right] B \\
d H / d t=\left(q E / E_{0}\right) M_{3}\left[1-\left(H / M_{3}\right)\right]-d_{3}\left[1-\left(E / E_{0}\right)\right] H \\
d E / d t=r E_{0}\left[1-\left(E / E_{0}\right)\right]-{ }_{S} D E
\end{gathered}
$$

The term $\left(E / E_{0}\right)$ in (3a), (3b) and (3c) accounts for how estrogen helps the growth of stem, tumor and health cells respectively. The parameter $E_{0}$ represents the carrying capacity or normal amount of Estrogen $E$. Further (i) If $E_{0}=E$, then the cells are dividing with normal rates, (ii) if $E_{0}>E$, then the cell are dividing with slower rates and (iii)
If $E_{0}<E$, then the cells are dividing with faster rates. Notations of the variables and parameters used in the model (3) are given in table 1. 
Table 1. Notations and representations of variables and parameters.

\begin{tabular}{ll}
\hline Notations & Representations \\
\hline $\mathrm{d}_{1,2,3}$ & Dying rates of cancer, tumors and health cells respectively due to the lack of sufficient amount of estrogen \\
$\mathrm{E}(\mathrm{t})$ & Amount of estrogen available to bind estrogen receptors in the cell \\
$\mathrm{k}$ & The rate of division of cancer stem cells \\
$\mathrm{E}_{0}$ & Normal amount of estrogen needed for all cells to divide normally \\
$\mathrm{M}_{1,2,3}$ & Carrying capacities of cancer stem cells, tumor cells and health cells respectively \\
$n$ & Death rate of cancer tumor cell \\
$\mathrm{q}$ & Normal rate of healthy cells near the tumor cells \\
$\mathrm{r}$ & Binding rate of estrogen with cells viz., cancer and health cells \\
$\mathrm{s}$ & Effectiveness $(0$ to 1$)$ of the Tamoxifen in blocking cells from receiving estrogen \\
$\mathrm{D}$ & Dose of Tamoxifen \\
\hline
\end{tabular}

\section{Simulation Study}

Tables 2. Parametric values used in figures.

\begin{tabular}{lllll}
\hline \multirow{2}{*}{ Parameter } & \multicolumn{4}{l}{ Parametric values used in } \\
\cline { 2 - 5 } & Figure 1 & Figure 2 & Figure 3 & Figure 4 \\
\hline$d_{1}$ & 0.50 & 0.40 & 0.55 & 0.40 \\
$d_{2}$ & 0.65 & 0.55 & 0.65 & 0.5 \\
$k$ & 0.20 & 0.20 & 0.20 & 0.20 \\
$E_{0}$ & 100 & 100 & 100 & 100 \\
$M_{1}$ & 20 & 20 & 20 & 20 \\
$n$ & 0.25 & 0.25 & 0.25 & 0.25 \\
$q$ & 0.75 & 0.75 & 0.75 & 0.75 \\
$r$ & 0.65 & 0.65 & 0.65 & 0.65 \\
$s$ & 0.60 & 0.85 & 0.45 & 0.25 \\
$D$ & 0 & 1 & 1 & 1 \\
$d_{3}$ & 0.1 & 0.1 & 0.1 & 0.65 \\
$M_{2}$ & 40 & 40 & 40 & 40 \\
$M_{3}$ & 60 & 60 & 60 & 60 \\
\hline
\end{tabular}

We now perform numerical simulations of the model (3) with various parametric values using MATLAB. The parametric values used in these simulations are given in Table 2

\subsection{Simulation Without Treatment}

We first simulate the model without treatment to visualize normal interactions between cancerous cells, healthy cells, and available estrogen.

Figure 1 shows that before the treatment (i) the number of cancer stem cells represented by blue curve increases with time from zero to the maximum carrying capacity following Brody growth function, (ii) the number of cancer tumor cells represented by red curve increases with time from zero to the maximum carrying capacity following Brody growth function, (iii) the number of health cells is normal (remains constant) and (iv) the amount of estrogen remains constant at the maximum level.

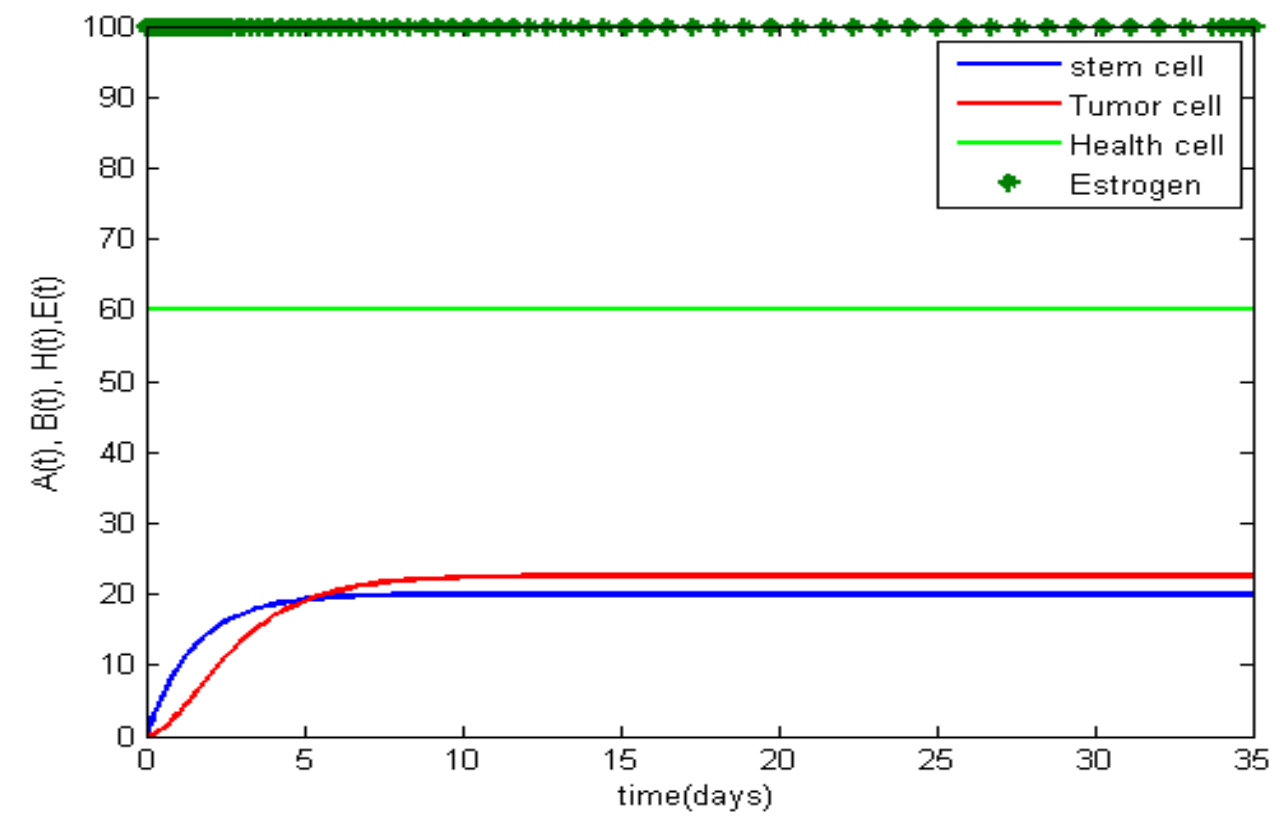

Figure 1. Brody growth model for breast cancer cells before treatment.

\subsection{Simulation with Treatment of Effectiveness $s=0.85$}

Recall that giving tamoxifen drug to a cancer patient is a kind of hormone therapy and the hormone therapy is a kind of treatment given to a cancer patient. The drug Tamoxifen is used for hormone therapy treatment. We now simulate the model (3) to show the effect of introducing treatment on all 
the three types the cells and on estrogen.

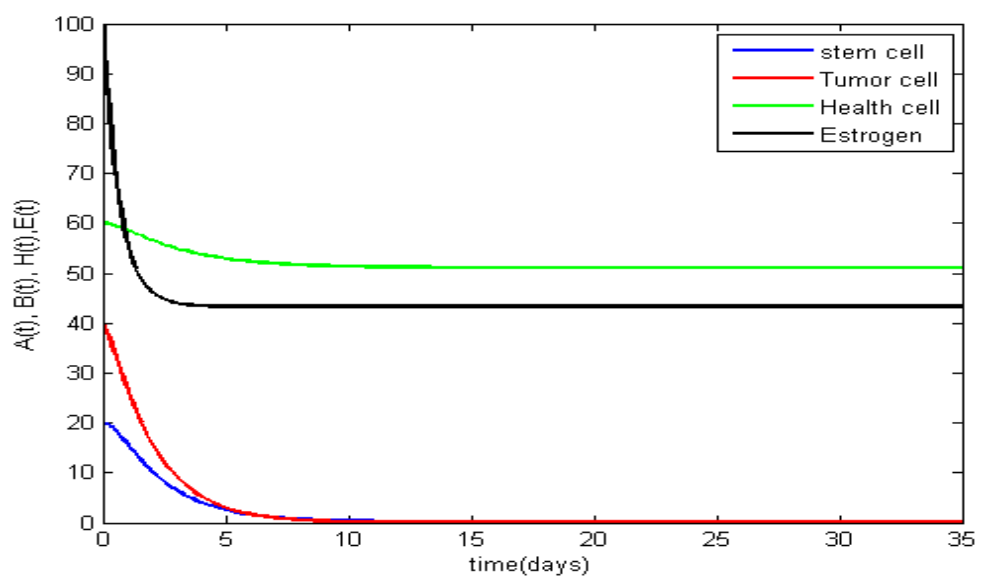

Figure 2. Brody growth model for breast cancer cells after treatment with effectiveness of $s=0.85$.

Figure 2 shows the affect of hormone therapy on all the three types of cells and on estrogen. Tamoxifin is given to meet an effectiveness of $s=0.85$. (i) The number of cancer stem cells represented by blue curve decreases with time from maximum carrying capacity 20 to zero following Brody growth function, (ii) the number of cancer tumor cells represented by red curve decreases with time from the maximum carrying capacity 40 to zero following Brody growth function, (iii) the number of health cells represented by green curve decreases with time from the maximum carrying capacity 60 to 55 following Brody growth function, and (iv) the amount of estrogen represented by black curve decreases with time from the maximum carrying capacity 100 to 43 following Brody growth function.

\subsection{Simulation with Treatment of Effectiveness $s=0.45$}

Figure 3 shows that the (i) amount of estrogen, (ii) growth of health cells and (iii) cancer cells coexist in the body of cancer patient when the treatment with an effectiveness of $\mathrm{s}=0.45$ is given. Since the sizes of both types of cancer cells viz., stem and tumor, decreased it may be possible to perform surgical treatment so as to nullyfy the cancer.

Further figure 3 shows the affect of hormone therapy on all the three types of cells and on estrogen. Tamoxifin is given to meet an effectiveness of $s=0.45$. (i) The number of cancer stem cells represented by blue curve decreases with time from maximum carrying capacity 20 to 10 following Brody growth function, (ii) the number of cancer tumor cells represented by red curve decreases with time from the maximum carrying capacity 40 to 3 following Brody growth function, (iii) the number of health cells represented by green curve decreases with time from the maximum carrying capacity 60 to 57 following Brody growth function, and (iv) the amount of estrogen represented by black curve decreases with time from the maximum carrying capacity 100 to 72 following Brody growth function.

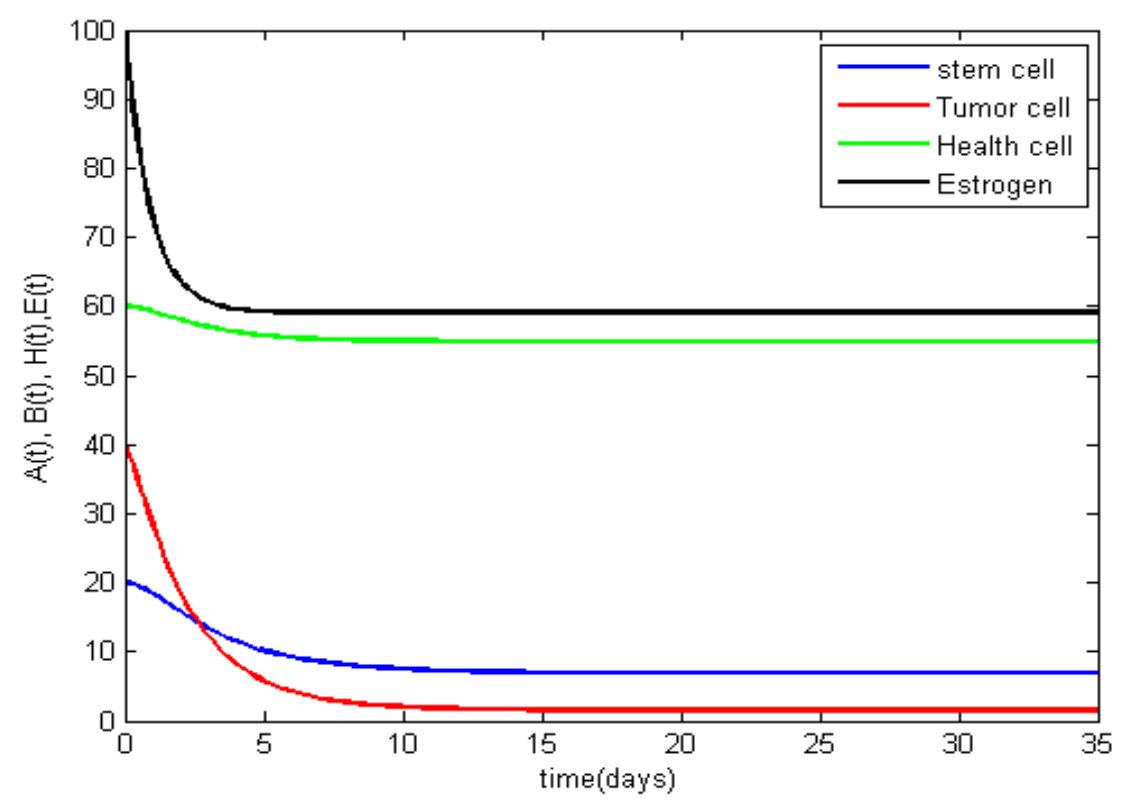

Figure 3. Brody growth model for breast cancer cells after treatment with effectiveness of $s=0.45$. 


\subsection{Simulation with Treatment of Effectiveness $s=0.25$}

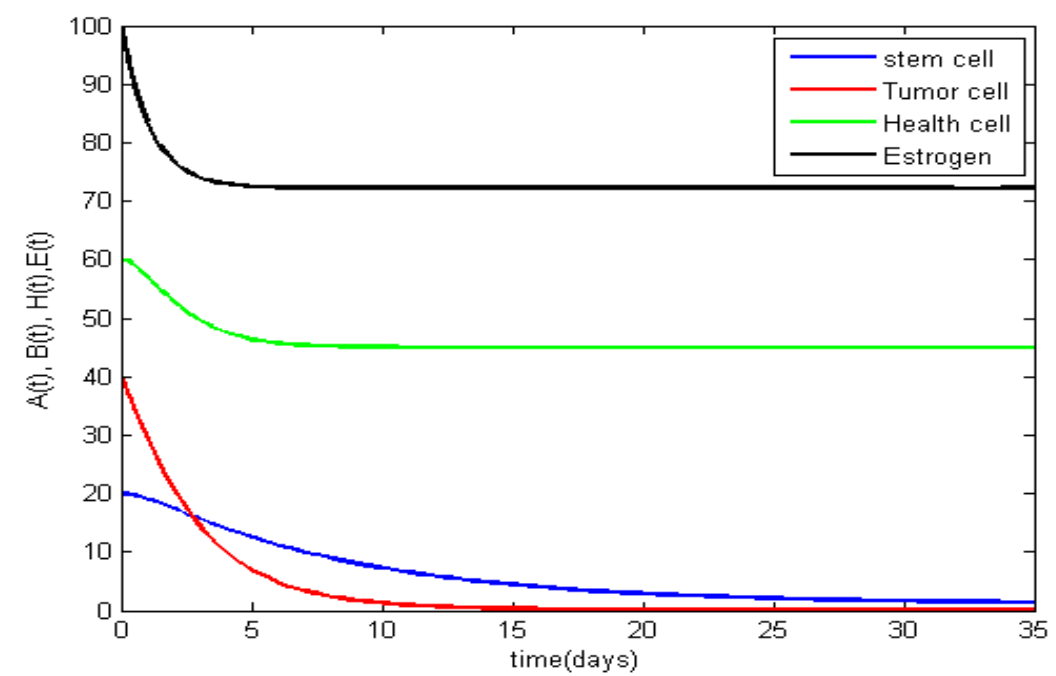

Figure 4. Brody growth model for breast cancer cells after treatment with effectiveness of $s=0.25$.

Further figure 4 shows the affect of hormone therapy on all the three types of cells and on estrogen. Tamoxifin is given to meet an effectiveness of $s=0.25$. (i) The number of cancer stem cells represented by blue curve decreases with time from maximum carrying capacity 20 to zero following Brody growth function, (ii) the number of cancer tumor cells represented by red curve decreases with time from the maximum carrying capacity 40 to zero following Brody growth function, (iii) the number of health cells represented by green curve decreases with time from the maximum carrying capacity 60 to 45 following Brody growth function, and (iv) the amount of estrogen represented by black curve decreases with time from the maximum carrying capacity 100 to 72 following Brody growth function.

Further in figure 4 it can be observed that the sizes of both cancer cells viz., tumor and stem, fall off to zero. However the fall of tumor cells is much faster than that of stem cells.

The observations made from the simulation study are tabulated below:

Table 3. Observations and suggestions of the simulation study.

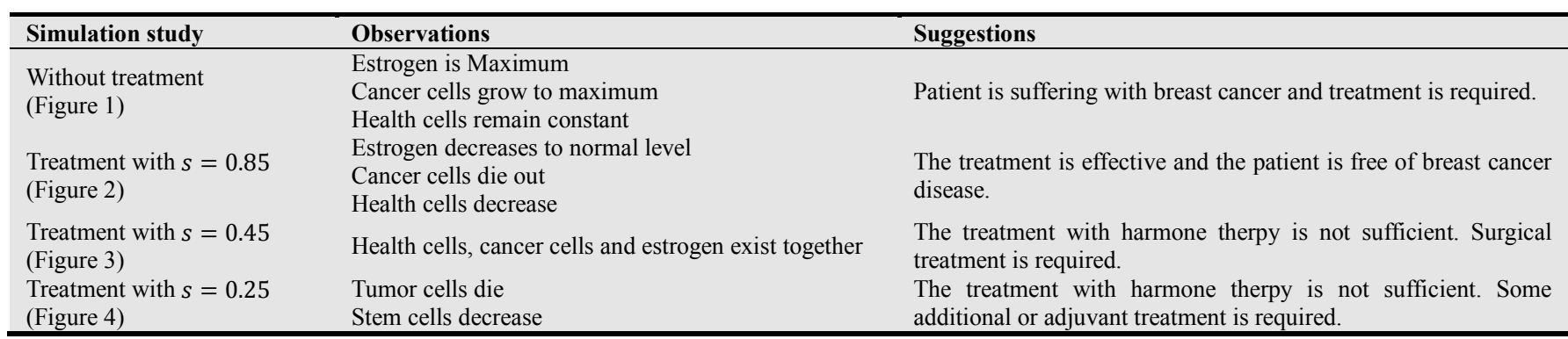

\section{Analysis of the Breast Cancer Model}

In this section we perform analysis of the breast cancer model designed using Brody function. The analysis includes (i) dimensionless form of the model, (ii) identifying equilibrium points, (iii) stability analysis of the equilibrium points, and (iv) positivity of equilibrium points. This analysis is required and of helpful to manipulate the parametric values in simulation study so as to obtain biologically meaning full results or conclusions.

\subsection{Dimension Less Form}

Dimensionless form of a mathematical model helps (i) to decrease the number of parameters involved in the model and (ii) to make the variables dimensionless, that is, the units of the variables are removed. Note that the minimization of the number of parameters and eliminating the units of variables will make the mathematical analysis simple. Hence, we construct the dimensionless form the model (3) in this section as follows:

We now introduce four dimensionless variables $x, y, z$ and $w$ defined by $x=\left(A / M_{1}\right), y=\left(B / M_{2}\right), z=$ $\left(H / M_{3}\right)$ and $w=\left(E / E_{0}\right)$ respectively. Also we introduce nine parameters $\mu, \delta_{i}, \zeta, \gamma, \sigma, \rho, t$ defined by $\mu=$ $\left(M_{1} / M_{2}\right), \delta_{i}=\left(d_{i} / k\right), i=1,2,3, \xi=(n / k), \gamma=(q / k)$, $\sigma=(s D / k), \rho=(r / k)$ and $\tau=\frac{t}{k}$ respectively. In view of introduction of these new variables and parameters the dimensionless form of the mathematical model (3) takes the form as follows: 


$$
\begin{aligned}
& \bar{x}=w(1-x)-\delta_{1}(1-w) x \\
& \bar{y}=w \mu x(1-y)-\xi y-\delta_{2}(1-w) y \\
& \bar{z}=w \gamma(1-z)-\delta_{3}(1-w) z \\
& \bar{w}=\rho(1-w)-\sigma w
\end{aligned}
$$

In (4), the over bar of the variables on the left hand side of each equation represent a differentiation with respect to $\tau$.

From the dimension less form of Brody model given in (4) we now identify the equilibrium points and undertake the stability analysis of these equilibrium points as follows:

\subsection{Equilibrium Points}

A point $x^{*}$ in real line $\mathfrak{R}$ is said to be an equilibrium point of one dimensional ordinary differential equation $x^{*}=f(x)$ if the point satisfies the condition $f\left(x^{*}\right)=0$, Equilibrium point can also be termed as stationary point or also as fixed point. Now in order to obtain the stationary point of the model (4) let us set the right hand sides of each of the four equations in (4) simultaneously equal to 0 and solve, so as to get the following:

$$
\begin{gathered}
x^{*}=\left[\rho /\left(\rho+\delta_{1} \sigma\right)\right] \\
y^{*}=\mu \rho^{2} /\left[\mu \rho^{2}+\xi(\sigma+\rho)\left(\rho+\delta_{1} \sigma\right)+\delta_{2} \sigma\left(\rho+\delta_{1} \sigma\right)\right] \\
z^{*}=\left[\gamma \rho /\left(\gamma \rho+\delta_{3} \sigma\right)\right] \\
J=\left[\begin{array}{cc}
-w-\delta_{1}(1-w) & (5 \mathrm{c}) \\
w \mu-w \mu y & -w x \mu-\zeta-\delta_{2}(1-w) \\
0 & 0 \\
0 & 0
\end{array}\right.
\end{gathered}
$$

$$
w^{*}=[\rho /(\rho+\sigma)]
$$

The dimensionless model (4) has the equilibrium point $P=\left(x^{*}, y^{*}, z^{*}, w^{*}\right)$ where the expressions for $x^{*}, y^{*}, z^{*}$ and $w^{*}$ are described in (5). We now take up the analysis of the stability of this equilibrium point $P=\left(x^{*}, y^{*}, z^{*}, w^{*}\right)$ by (i) constructing the Jacobian matrix and (ii) finding the eigenvalues of the Jacobian matrix and (iii) finding the signs of these Eigen values at the equilibrium point.

\subsection{Positivity of Equilibrium Point}

In order to show that the model (4) is well defined and is biological meaning full, we restrict that all of the four population sizes can approach a non-negative steady state. Hence, we establish the condition on the parameters as $\mathbb{R}_{+}^{4}=\{(x, y, z, w): x, y, z, w \geq 0\}$. These non-negativity conditions are required to be satisfied for the equilibrium points. To guarantee the $x, y, z, w \geq 0$ for all value of the parameters $\mu, \delta_{i}, i=1,2,3, \xi, \gamma, \sigma, \rho, \tau \geq 0$ found in the dimension less form is positive hence the mathematical model of Brody growth model for breast cancer is well defined both mathematical and biological.

\subsection{Stability Analysis of the Equilibrium Points}

The Jacobian matrix is used to analyze the local stability of equilibrium points where the Jacobian matrix for the model (4) is given by

$$
\begin{aligned}
& 1-x+\delta_{1} x \\
& x \mu(1-y)+\delta_{2} y \\
& \begin{array}{c}
0 \\
-w \gamma-\delta_{3}(1-w) \\
0
\end{array} \\
& \left.\begin{array}{c}
\gamma(1-z)+\delta_{3} z \\
-\sigma-\rho
\end{array}\right] \\
& \begin{array}{c}
\lambda_{1}=-\left[\left(\rho+\delta_{1} \sigma\right) /(\sigma+\rho)\right] \\
\lambda_{2}=R_{2} \\
\lambda_{3}=-\left[\left(\rho+\delta_{3} \sigma\right) /(\sigma+\rho)\right] \\
\lambda_{4}=-(\rho+\sigma)
\end{array}
\end{aligned}
$$

Based on the algebraic sign of the Eigen values $\lambda_{1}, \lambda_{2}, \lambda_{3}$ and $\lambda_{4}$ of the Jacobian matrix, we can determine the nature of the equilibrium points. Since all the Eigen values are less than zero (negative) the dimensionless system(4) described using Brody growth function is stable at the equilibrium point $P=\left(x^{*}, y^{*}, z^{*}, w^{*}\right)$. the following:

$$
\begin{gathered}
R_{1}=\frac{\rho \mu\left(\zeta(\rho+\sigma)\left(\rho+\delta_{1} \sigma\right)+\delta_{2} \sigma\left(\rho+\delta_{1} \sigma\right)\right)}{(\rho+\sigma)\left(\rho^{2} \mu+\zeta(\rho+\sigma)\left(\rho+\delta_{1} \sigma\right)+\delta_{2} \sigma\left(\rho+\delta_{1} \sigma\right)\right)} \\
R_{2}=-\frac{\left(\rho^{2} \mu+\zeta(\rho+\sigma)\left(\rho+\delta_{1} \sigma\right)+\sigma\left(\rho+\delta_{1} \sigma\right)\right)}{(\rho+\sigma)\left(\rho+\delta_{1} \sigma\right)} \\
R_{3}=\frac{\rho \mu}{\rho+\delta_{1}}\left(\frac{\zeta(\rho+\sigma)\left(\rho+\delta_{1} \sigma\right)+\delta_{2} \sigma\left(\rho+\delta_{1} \sigma\right)+\delta_{2}\left(\rho^{2} \mu\right)}{\left(\rho^{2} \mu\right)+\zeta(\rho+\sigma)\left(\rho+\delta_{1} \sigma\right)+\delta_{2} \sigma\left(\rho+\delta_{1}\right)}\right)
\end{gathered}
$$

\section{Conclusions}

Mathematical modeling has been an ever increasing tool to explore complex systems and unsolved problems in biology so as to make the study of their behavior simple. In this paper, we have applied Brody growth function on the mathematical modeling of growth of breast cancer cells and response of hormone therapy on cancer disease. The Brody growth function is a special case of Koya-Goshu generalized model and the former is a better model to address the breast cancer cell growth. In this study we have considered the linear

The four Eigen values of the Jaco
at the equilibrium point are given by 
network growth of cancer cells and also the growth of all cells depend on estrogens.

The maximum levels of estrogen in the human body results in (i) making the abnormal growth of health cells by protecting them from the natural death and (ii) occurrence of estrogen positive respiratory breast cancer and its rapid growth (see Figure 1). When hormone therapy is given to a breast cancer patient it is observed that (i) the estrogen level is reduced rapidely to the normal size and (ii) the tumor and stem cells die together. Hence, the hormone therapy tretements is effectiveon breast cancer (see Figure 2). Even though the health cells, cancer stem and tumor cells and estrogen present in the body of breast cancer patient, if the cancer cell size is reduced to a minimum level surgery can be performed to cure the cancer. The simulation study supports such a reduction of cancer cells as displayed in Figure 3.Thesimulation study in figure 4 show that all cancer tumor cells are died,cancer stem cells are reduced in size but not compeltly removed, health cells are reduced to the normal size, and estrogen is rapidely decreased to normal size. Thus, in this case, an adjuvant treatment i.e., an additive that enhances the effectiveness of medical treatment, is required.

Further, the simulation study supports that the application of brody growthfunction to breast cancer model is biological meaining full and matematical well defined. The model constructed and studied in this paper is local stable at the equlibruim point.

\section{References}

[1] J.D. Murray, Mathematical Biology, Interdisciplinary Applied Mathematics, 2002.

[2] Chipo Mufudza, Walter Sorofa, and Edward T. Chiyaka, Assessing the effects of Estrogen on the Dynamics of Breast Cancer, Hindawi publishing Corporation,Vol.2012 Article ID473572,14pages,doi:10.1155/2012/473572.

[3] Tesfay Hailu, Hailemariam Berhe, Knowledge of Breast Cancer and its Early detection measures among Female Students in Mekelle University, Tigray region, Ethiopia,2014,p.57-64.

[4] Purnachandra Rao Koya, Ayele Taye Goshu. Generalized Mathematical Model for Biological Growths, Open Journal of
Modelling and Simulation. Vol. 1, 2013, pp. 42-53. http://dx.doi.org/10.4236/ojmsi.2013.14008

[5] Tsygvintsev, Alexei and Marino, Simeone and Kirschner, Denise E, A Mathematical Model of Gene Therapy for the Treatment of Cancer, Mathematical Methods and Models in Biomedicine, pp.367-385, 2013.

[6] Gebremedhin A and Shamebo M., Clinical Profile of Ethiopian Patients with Breast Cancer, East African Medical Journal,Vol.75, No.11, pp.640-643, 1998.

[7] Rachel Roe-Dale, David. I, Michael. K. Â, A Mathematical Model of Breast Cancer Treatment with CMF and Doxorubicin, 2010

[8] Eric Werner, Cancer Networks: A general Theoretical and Computational Framework for Understanding Cancer, arXiv:1110.5865v1 [q-bio.MN] 26 Oct 2011,University of Oxford.

[9] Semarya B., Worknish, Mignote H., MesfinA., Alemseged A., Assessment of Knowledge of Breast Cancer and Screening Methods among Nurses in University Hospitals in Addis Ababa, Ethiopia, 2011.

[10] Michelle McDuffie, A Hormone Therapy Model for Breast Cancer Using Linear Cancer Networks, Volume 15, No. 1, Spring 2014.

[11] HaftomGebrehiwot1, Tesfay Hailu, Gebreamlak Gidey, Knowledge and Attitude towards Breast Cancer among Mekelle University, Female Regular Undergraduate Students, Tigray Region, Ethiopia, 2013 ,ISSN 2320-6691 (Online)

[12] Tang, Betty and Wolkowicz, Gail SK, Mathematical Models of Microbial Growth and Competition in the Chemostat Regulated by cell-bound Extracellular Enzymes, Journal of Mathematical Biology, Vol. 31, No. 1, pp. 1-23, 1992, Springer.

[13] Ferlay, Jacques and Hery, Clarisse and Autier, Philippe and Sankaranarayanan, Rengaswamy, Breast Cancer Epidemiology, pp.1-19,Spring 2010,

[14] Source: Ferlay J, Soerjomataram I., Ervik M., Dikshit R., Eser S., Mathers C., Rebelo M., Parkin D. M., Forman D., Bray, F.GLOBOCAN 2012 v1.1, Cancer Incidence and Mortality Worldwide: IARC Cancer Base No. 11 [Internet]. Lyon, France: International Agency for Research on Cancer; 2014. Available from: http://globocan.iarc.fr, accessed on 16/01/2015. 